Thorax (1951), 6, 408

\title{
BRONCHOGRAPHY IN CHILDREN
}

\author{
BY \\ M. BATES, I. C. W. ENGLISH, H. M. FOREMAN, AND T. M. WILSON \\ From the Brompton Hospital, London
}

(RECEIVED FOR PUBLICATION SEPTEMBER 11, 1951)

To produce satisfactory bronchograms in children is generally considered difficult. Most children under the age of 12 years require a general anaesthetic before the procedure is possible, and in the past this has often detracted from the quality of the bronchograms.

We have gradually evolved a technique using general anaesthesia by which consistently satisfactory bronchograms are obtained. The method is safe, free from complications, and causes the minimum of physical or mental upset to the child.

\section{TECHNIQUES}

Techniques for bronchography in children can be divided into those done under (1) local anaesthesia, (2) general anaesthesia, and (3) a combination of local and general anaesthesia.

(1) Methods using Local Anaesthesia.-The opaque oil is instilled through the nose after the nose, pharynx, and larynx have been anaesthetized. The co-operation of the child is essential, and is difficult to obtain. One cannot ensure in any case that the oil will pass into the trachea, and the dosage of oil for each lobe is therefore difficult to judge. Another disadvantage is that the child has an anaesthetized larynx for about two and a half hours after the operation, and eating and drinking have to be guarded against during that period. A needle is passed into the trachea through the crico-thyroid membrane under local anaesthesia. The use of a needle exposes the child to a risk of infection through the puncture and surgical emphysema, locally or in the mediastinum. The insertion of a needle is a frightening procedure, and the co-operation of the child is therefore doubly difficult to obtain.

If the child is co-operative these techniques have the advantage of being less complicated, no skilled anaesthetist is needed, and the child can voluntarily stop breathing when the radiographs are to be taken.

(2) Methods under General Anaesthesia.-These me:hods usually rely on complete relaxation under deep anaesthesia, which often implies heavy preliminary sedation, for example, with avertin, and a long post-operative recovery period.

Jacoby and Keats (1938) use deep anaesthesia with open ether and instil the opaque oil into the pharynx so that it will run over from the pyriform fossae into the larynx and trachea. The authors themselves say that occasionally bronchography has to be abandoned because of cough and excess bronchial secretion. We think that the deficiencies of this method are the lack of direct control of the airway, no assurance that the oil will pass into the trachea, and no provision for removing 
excessive secretions or oil. If deep anaesthesia is to be produced the little further effort of passing an endotracheal tube seems well worth while.

Barsby and Bonham-Carter (1941) use a similar method employing deep general anaesthesia, but injecting the oil directly over the larynx with a laryngeal syringe. This method has the same drawbacks. Pearson and Thornton (1939) describe a method essentially the same as that used in this hospital for many years, that is, passing an endotracheal tube under deep anaesthesia and running the opaque oil down that tube. The oil can temporarily block the small internal diameter of the endotracheal tube. These authors make no mention of bronchial suction or of controlled or assisted respiration. In our experience this method was precarious, as the child could maintain his airway only by sucking oil down the tube ; the exact quantity of oil reaching the carina could not be assessed, and the inability to control respiration made it difficult to take a radiograph free from respiratory movement.

(3) Methods using Combined Local and General Anaesthesia.-Lyons (1939) described a method closely resembling our own, but using a nasal endotracheal tube after preliminary nasal, pharyngeal, and laryngeal local anaesthesia. The routine use of a nasal endotracheal tube limits the size of the tube which can be used, and it may also cause nasal sinus infection. The local anaesthesia of the larynx in addition to the general anaesthesia may mean that the cough reflex will not be present if the child vomits during recovery from general anaesthesia, and therefore the vomit may be inhaled. His practice of inserting a catheter into each main bronchus is unnecessary if satisfactory posturing is used, and makes the method unduly complicated.

In 10 cases we tried transitory general anaesthesia, combined with local anaesthesia, and the insertion of a needle through the crico-thyroid membrane as described by Field (1942). We found that this method was an ordeal both for the child and for the operator. Recovery from general anaesthesia to find a needle in the neck causes alarm and discomfort in most children. Furthermore, it is very difficult to prevent coughing, vomiting, or at least retching, during the recovery period, and any of these is likely to dislodge the needle from the trachea. The protests of the child and his lack of further co-operation may make it impossible to proceed.

The risks of inserting a needle into the trachea have already been mentioned. Field reports two complications in 100 cases. However, with this method if all goes smoothly good pictures are obtained.

Bronchograms are assessed on the completeness of bronchial filling and the clarity of the picture. It is not possible to judge the efficiency of the methods described by the various authors we have quoted, as none gives an analysis of the quality of the bronchograms obtained by their method of choice.

\section{Development of Present Method}

In evolving our present method we have experimented with various techniques during the past five years in over 250 cases. At first we followed a method similar to that described by Pearson and Thornton (1939) which had been used in this hospital for some years. We overcame what were considered the deficiencies of this method by using lighter anaesthesia and delivering the oil near the carina.

We tried anaesthetizing with cyclopropane, with pentothal, curare, and nitrous oxide, and with nitrous oxide, oxygen, and ether, and found that ether produced the most satisfactory bronchial dilatation and results. 


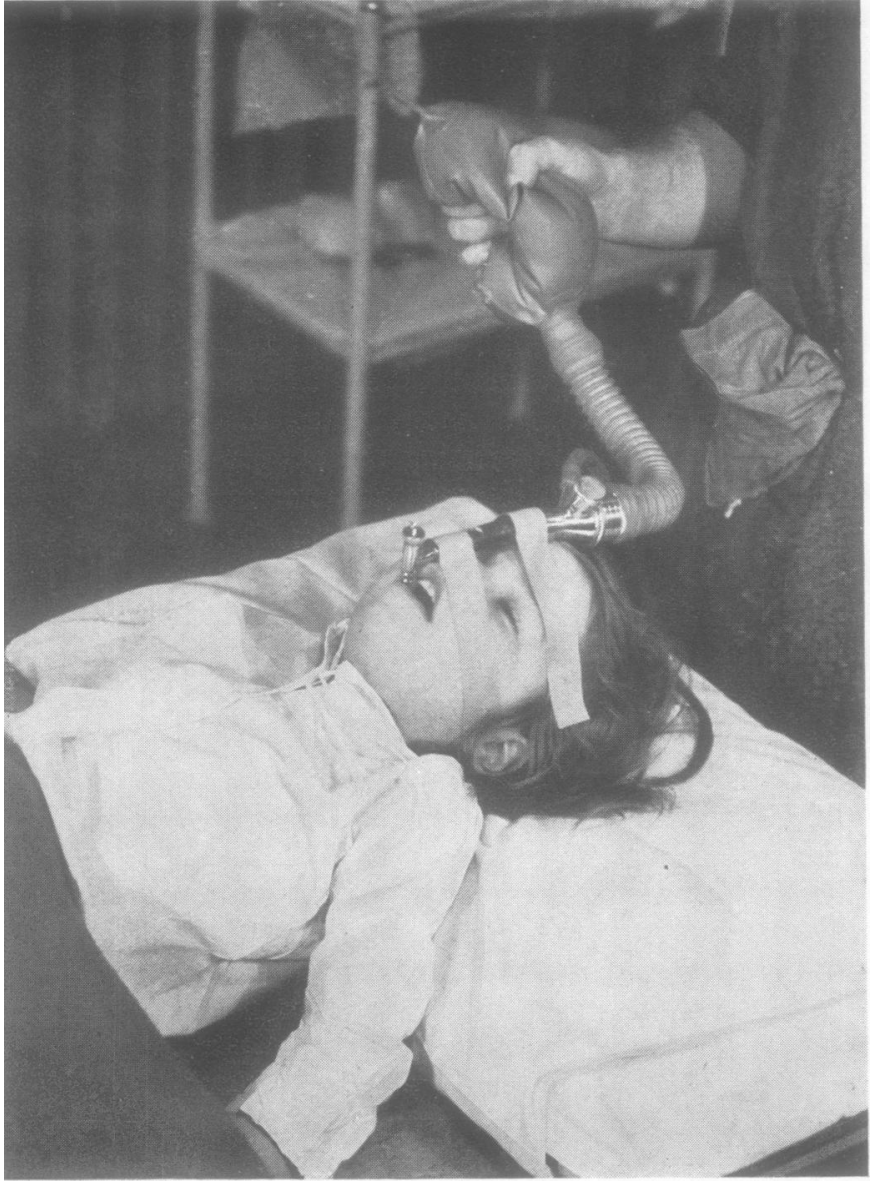

FIG. 1.
To ensure that the required volume of oil was delivered just above the carina we used a small bore gum-elastic urethral catheter reaching to the tip of the endotracheal tube. This modification also ensured that the endotracheal tube was not obstructed by oil.

Some operators give a single injection of oil for each lung and then quickly posture for the various lobes in that lung. We had better results by giving separate injections of oil for each lobe with the patient in the appropriate posture for that lobe (Figs. 1-5).

On occasion we failed to fill portions of the bronchial tree when all the necessary conditions seemed to be present, and therefore tried warming the opaque oil to make it less viscous. However, the increased tendency to alveolar filling made us discard this modification and we now believe that the viscosity of the oil before injection has no relation to inadequate filling.

We investigated whether the use of controlled respiration predisposed to alveolar filling, but compared series showed that this was not so. However, we think that bronchospasm can cause either inadequate bronchial or excessive alveolar filling. The filling is inadequate if the constricted bronchi contain even a small plug of mucus ; it is excessive if the oil is carried past the constriction by the increased rate of flow of anaesthetic gases necessary to maintain adequate respiratory exchange in the presence of bronchospasm. Whatever anaesthetic agent is used, it is essential to produce regular, shallow respiration, and this we consider the most important single factor in producing a satisfactory bronchogram. If this type of respiration can be obtained by relatively light anaesthesia this is preferable to the long-acting agents which produce unnecessary depression post-operatively.

Other important factors for satisfactory bronchography are correct positioning of the patient and speed. Not more than a minute (for example, 15 seconds each lobe) should be taken to complete the filling of one lung, otherwise alveolar filling is likely to occur.

Respiratory movement must be eliminated when the pictures are taken.

A good radiographic technique must be used. 


\section{Details of Method}

After admission to the ward the child's condition is assessed. Should he have fever or be producing a large quantity of sputum, bronchography is postponed.

Preparation.-Repeated postural drainage is given. Usually the child is already being taught breathing exercises. If necessary chemotherapy or treatment with antibiotics is arranged.

Premedication.-The child is promedicated with atropine, 1/100 gr., and omnopon in a dosage of 1/30 gr. per stone body weight one hour before starting the anaesthetic.

Anaesthesia.-An induction with cyclopropane and oxygen is used for speed and smoothness. Any coughing will tend to loosen sputum which at that stage cannot be removed. Using a laryngoscope, the child is intubated as soon as possible with the largest endotracheal Magill tube that can easily be passed (Fig. 1). Anaesthesia is continued with nitrous oxide oxygen and ether, any secretions being aspirated at once. A little initial overventilation soon produces a state of acapnia and controlled breathing can be maintained until after the pictures have been taken. It is obvious from the feel of the " bag" if any bronchospasm is present and it is quite useless to start injecting oil until this has entirely disappeared. Ether always abolishes it sooner or later without the plane of anaesthesia necessarily being deep. When bronchoscopy is to be done under the same anaesthetic an induction with pentothal and curare is used, followed for the bronchography by gas, oxygen, and a little ether as before.

Injection of Oil.- The length of the endotracheal tube has been marked, pərhaps with zinc oxide plaster, on a fine gum-elastic suction catheter, size 8 . The catheter is then

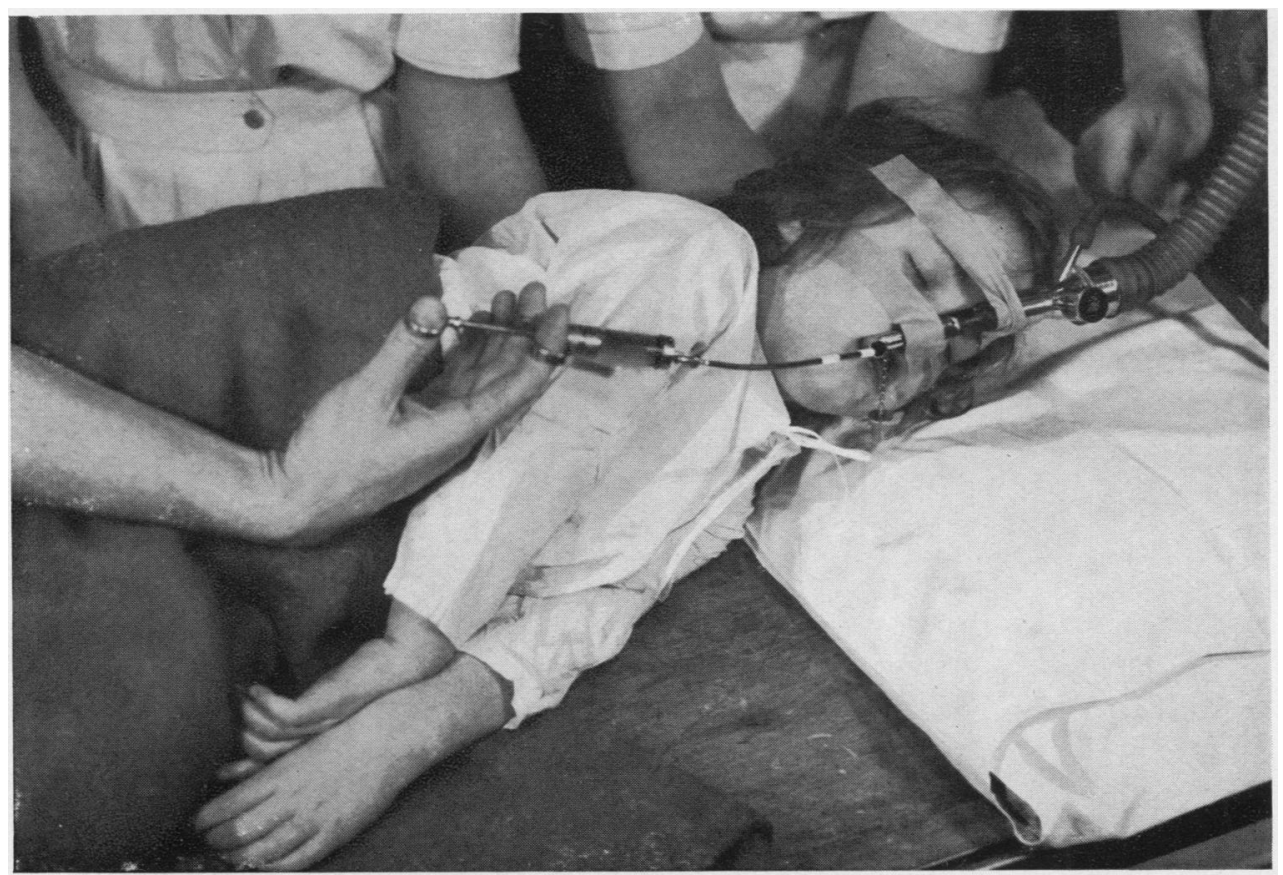

FiG. 2. 


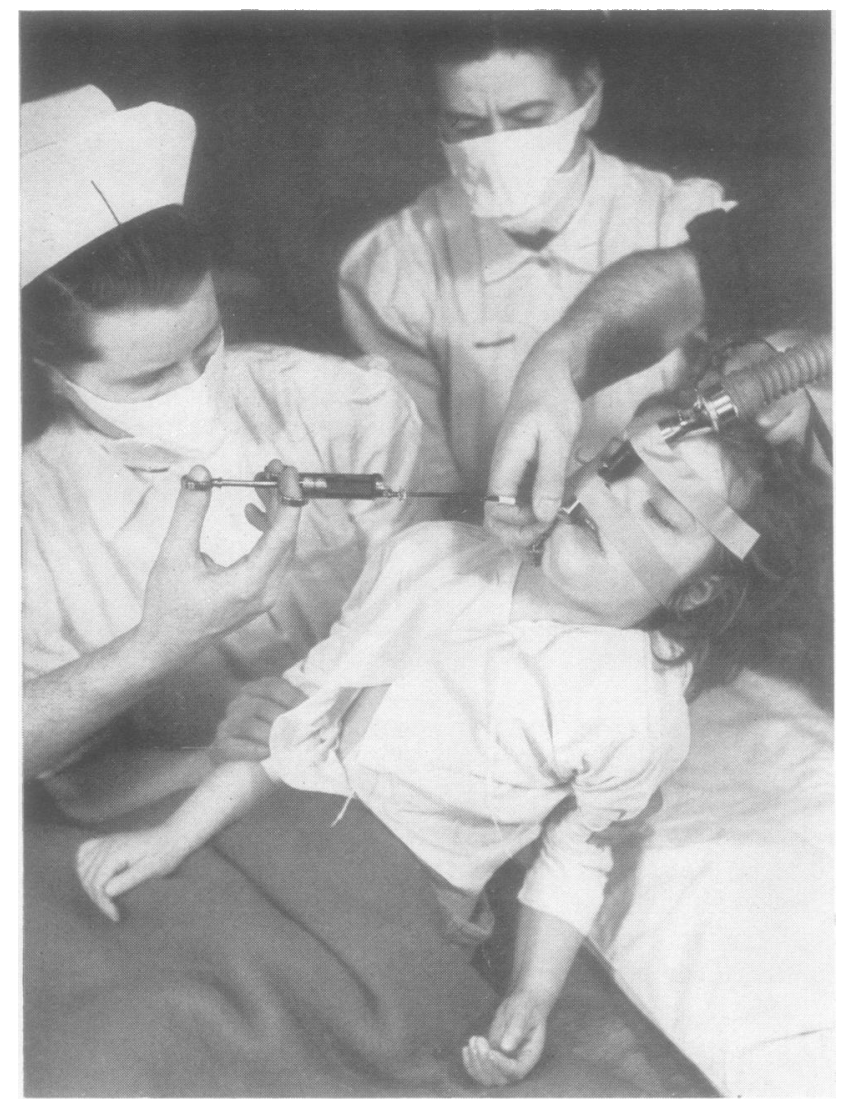

FIG. 3.

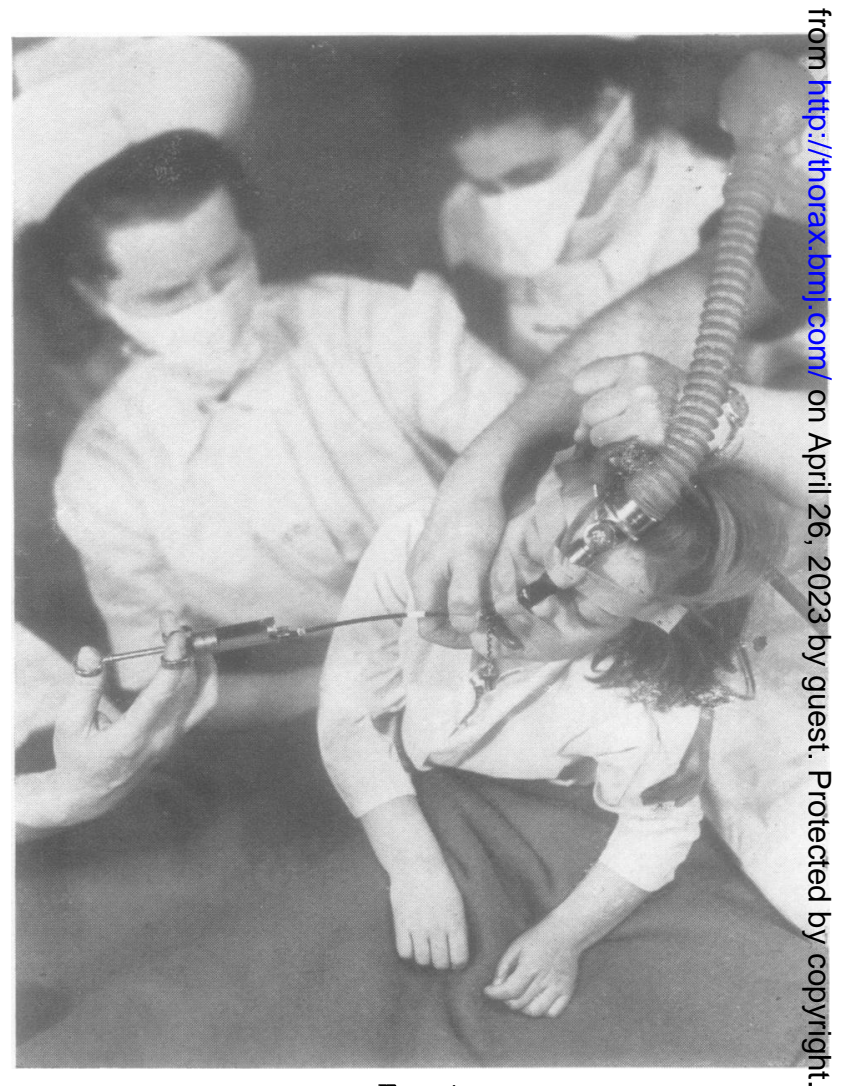

FIG. 4. 


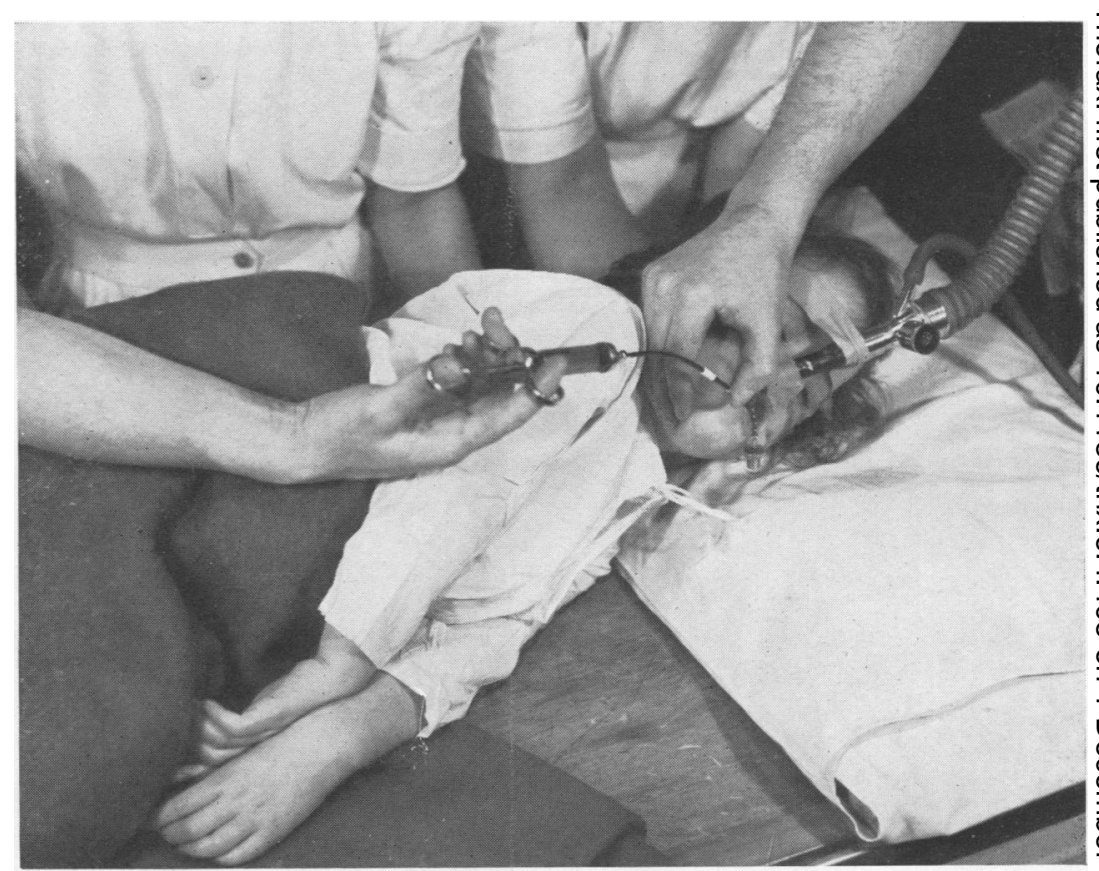

FIG. 5.

introduced inside the endotracheal tube to the distance marked (Fig. 2) so that the oil will be delivered at the end of the endotracheal tube. The oil is injected down the gumelastic catheter with an ordinary 10 or $20 \mathrm{ml}$. Labat syringe.

We have found the fractional method of injecting each lobe in turn the most satisfactory, if the appropriate posture for each lobe is strictly carried out. To allow controlled breathing to continue during the injection of the oil the anaesthetist's thumb blocks the hole in the suction T-piece.

Dosage of Opaque Oil.-The approximate amount of oil needed for each lung is $1 \mathrm{mi}$. per year of apparent age up to $5 \mathrm{ml}$.; for example, $3 \mathrm{ml}$. each side for a child of 3 -year size. We have found $5 \mathrm{ml}$. to be the maximum required for children up to the age of 12 years. Usually the injection is subdivided into lower lobe $3 \mathrm{ml}$., middle lobe $1 \mathrm{ml}$., upper lobe $1 \mathrm{ml}$.

Posturing.-If bilateral bronchograms are required the right lung is filled first, appropriate radiographs taken, and then the left lung filled. We have done bronchograms on two children with situs inversus; the order should then of course be reversed.

The patient is postured as shown in Figs. 3, 4, and 5, being kept in each appropriate posture for not more than 15 seconds, during which time the required quantity of oil is injected. If the oil is heard bubbling in the trachea or main bronchus, or the airway impaired, the child should be sat upright for a few seconds.

For satisfactory posturing team-work is essential and requires a nurse, operator, radiographer, and anaesthetist. The nurse holds the child's head in line with the spine and the radiographer holds and postures the child, making certain that the thoracic spine is kept straight. If the thoracic spine is allowed to curve laterally, particularly in the half upright position used for lower lobe filling, oil will run into the upper lobe bronchus. Therefore, in posturing for the lower lobe the child should be rotated to the appropriate side around the axis of the thoracic spine and not leant to that side. The upper lobe is easily filled even when no Trendelenburg position is used.

\section{RADIOGRAPHY}

The radiographs should be taken as quickly as possible after filling has been completed. The flow of ether vapour is turned off before the $x$-ray apparatus is used. 
FIG. 7.

Figs. 6 AND 7 -To illustrate adeauate and inadequate filling. Fig. 6 is adequate. but Fig. 7 is unacceptable because of poor basal filling. 
If bilateral bronchograms are being done the right lung is filled first and the order of taking radiographs is :

Right Lung: Right lateral view.
Antero-posterior view.

Left Lung: Right anterior oblique view. (Patient rotated half on to left side with left shoulder on film.)

Antero-posterior view.

In this way good views of both bronchial trees are obtained, as described by FosterCarter (1943). If a left bronchogram alone is required, a left lateral view can be taken in addition. If on viewing the wet plates the filling is seen to be unsatisfactory, a further attempt may be made to fill the required area, but in our experience second attempts under the same anaesthetic are seldom successful.

Recovery.-When the radiographs have been seen and passed as satisfactory the mixture of opaque oil and bronchial secretion is aspirated from the bronchial tree by passing a suction catheter down the endotracheal tube. The endotracheal tube is removed as soon as the patient has recovered his reflexes and replaced by an ordinary airway, and the child is then returned to the ward.

\section{Analysis of Bronchographic Results}

We have not found a paper in which the results have been judged statistically. The methods advocated by different authors can be assessed only if the results are analysed in detail.

The method described has been used in about 250 cases in children, but the records of only 120 remain for analysis after a recent fire. We submitted these 120 bronchograms to an independent radiologist for evaluation.

A bronchogram was considered satisfactory only if (1) all segmental bronchi were outlined ; (2) bronchial outlines were clear and not obscured by alveolar filling ; and (3) radiological technique was adequate (e.g., no movement and correct exposure for contrast).

The analysis of the results in these 120 cases is as follows.

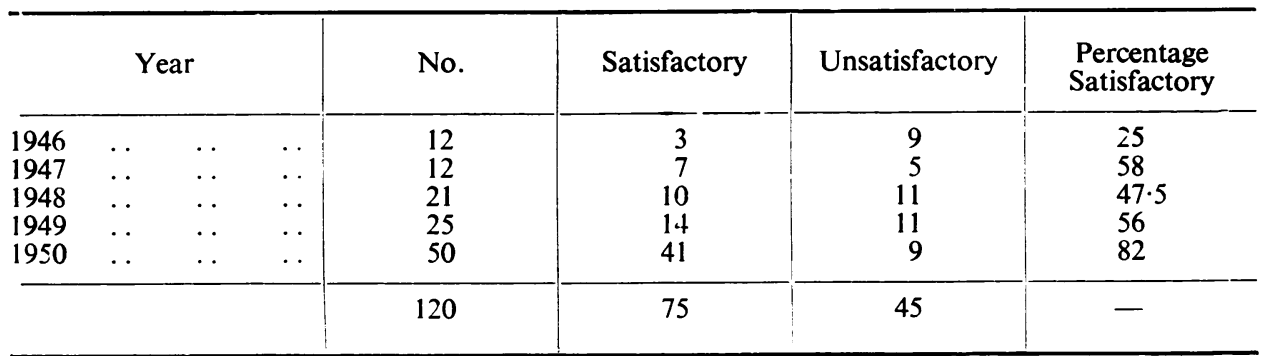

The progressive improvement in our results is obvious, although even the latest percentage of satisfactory results may seem poor in comparison with the nonstatistical impressions previously published.

We now feel confident that we can produce satisfactory bronchograms in at least $80 \%$ of attempts.

It is emphasized that a bronchogram is considered satisfactory only if there is complete filling of all segmental bronchi as is required for surgical assessment (Figs. 6 and 7). 


\section{SUMMARY}

A method for bronchography in children is described. This method has been used in 250 cases without complications. Satisfactory results are now obtained in at least $80 \%$ of attempts.

\section{REFERENCES}

Barsby, B. E., and Bonham-Carter, R. E. (1941). Arch. Dis. Childh., 16, 95.

Field, C. E. (1942). Lancet, 2, 357.

Foster-Cartar, A. F. (1943). Proc. roy. Soc. Med., 36, 451.

Jacoby, N. M., and Keats, G. (1938). Lancet, 2, 191.

Lyons, S. S. (1939). Curr. Res. Anesth., 18, 168.

Pearson, H. E. S., and Thornton, H. L. (1939). Brit. J. Radiol., 12, 229.

\section{AdDENDUM}

While this paper was being prepared and written we had no complications in these patients due either to the anaesthetic or to the bronchogram. But recently a serious complication occurred in a boy aged 7. After the radiographs had been taken the heart stopped beating, presumably from vagal stimulation, and it was 10 minutes before a satisfactory circulation was re-established. The child recovered, in so far as he is still alive, but four months after the bronchogram was made he is in a state of complete decerebrate rigidity. 